\title{
Teorías subjetivas docentes acerca de la inclusión en contexto de pandemia
}

\author{
Subjective Theories of Teachers About Inclusion in the Context of a Pandemic
}

\author{
Janett Yañez-Collado ${ }^{1}$; Carola Cerpa-Reyes ${ }^{2}$
}

\begin{abstract}
RESUMEN
El presente artículo da cuenta de una investigación acerca de las teorías subjetivas sobre inclusión escolar expresadas por docentes que se desempeñan en modalidad remota, en el actual contexto de pandemia por COVID-19. Se utilizó un enfoque cualitativo de carácter descriptivo-interpretativo, enmarcado en la grounded theory. Participaron en la investigación ocho docentes, pertenecientes a un colegio particular-subvencionado de educación básica y media, ubicado en la comuna de Coquimbo, Chile. El instrumento de recolección utilizado fue la entrevista en profundidad y los procesos de análisis de contenido corresponden a las fases de codificación abierta, axial y selectiva. Las teorías subjetivas docentes respecto a la inclusión en el contexto no presencial se centran en la dificultad para garantizar el acceso y la participación de todos/as los/as estudiantes, lo que incide en la exclusión de los/as más vulnerables. En relación al desempeño docente, queda de manifiesto el gran desafío que significó para el profesorado adecuarse al actual modelo educativo. No obstante, quienes participaron en este estudio consideran que la adquisición de conocimientos digitales favorece el ejercicio de su práctica educativa y que las metodologías basadas en recursos digitales permanecerán y se afianzarán en las clases presenciales, contribuyendo al mejoramiento del aprendizaje de los estudiantes.
\end{abstract}

Palabras claves: inclusión; necesidades educativas especiales; educación no presencial; teorías subjetivas; pandemia; COVID-19.

\begin{abstract}
This article reports on an investigation about subjective theories on school inclusion expressed by teachers who work remotely, in the current context of the COVID-19 pandemic. A qualitative, descriptive-interpretive approach was used, framed in grounded theory. Eight teachers participated in the research, belonging to a private-subsidized school of basic and secondary education, located in the

\footnotetext{
${ }^{1}$ Educadora diferencial y coordinadora PIE en Colegio Santa María de Belén, Coquimbo, Chile; magíster en Inclusión, Universidad Central, Chile; janettyanez23@gmail.com.

${ }^{2}$ Académica de la Facultad de Ciencias Sociales, Universidad Central, Coquimbo, Chile; psicóloga, magíster en Psicología Educacional; cacerpa@gmail.com; https://orcid.org/0000-0002-6180-5262.
} 
commune of Coquimbo, Chile. The collection instrument used was the in-depth interview and the content analysis processes correspond to the open, axial and selective coding phases. The subjective teaching theories regarding inclusion in the non-face-to-face context focus on the difficulty of guaranteeing access and participation for all students, which affects the exclusion of the most vulnerable. In relation to teaching performance, it is evident that teachers faced great challenges to adapt to the current educational model. However, those who participated in this study consider that the acquisition of digital knowledge favors the exercise of their educational practice, and that methodologies based on digital resources will remain and be consolidated in face-to-face classes, contributing to the improvement of student learning.

Keywords: Inclusion; special educational needs; non-face-to-face education; subjective theories; pandemic; COVID-19.

A nivel mundial, la pandemia de COVID-19 ha impactado profundamente todos los ámbitos de la vida social, lo que ha obligado a las personas a introducir cambios en sus conductas a fin de afrontarla de la mejor manera posible. En Chile se ha implementado un estado de emergencia y confinamiento de la población, lo cual ha requerido la instalación de un modelo educativo no presencial. El Ministerio de Educación (Mineduc, 2020a) ha dispuesto un conjunto de acciones para mantener la continuidad educativa, siendo la educación remota mediada por tecnologías el principal recurso para suplir la presencialidad. El Mineduc (2020b) entiende que los y las docentes deben asumir grandes retos en el escenario actual, para lograr que sus estudiantes puedan cumplir con los objetivos de aprendizaje y desarrollo planificados para este periodo.

Los desafíos que impone el nuevo escenario educativo han provocado agobio laboral en los y las docentes, dadas las nuevas exigencias respecto a las actividades que han debido implementar de forma autónoma. Ha quedado de manifiesto "la necesidad de una formación docente en educación a distancia, que va más allá de la acción emergente, con una preparación para comprender el funcionamiento que implica el trabajo modular y el uso de nuevas plataformas digitales" (Garrido, 2020, p.58).

Según datos entregados por la OCDE (Organización para la Cooperación y el Desarrollo Económicos, 2019), el 12,5\% de las/os estudiantes en Chile no cuenta con acceso a internet. Si bien la tasa de conectividad es alta en comparación con otros países, la estabilidad de la conexión no se encuentra plenamente asegurada. Además, Chile aparece como el segundo país con menor 
diversidad en el acceso, registrando una de las más altas tasas de desigualdad. Las familias de menores ingresos son las que menos posibilidades tienen de acceder a este servicio y, si lo obtienen, este es de inferior calidad (OCDE, 2019). Se profundiza así la desigualdad educativa, debido a que un gran porcentaje de estudiantes no cuenta con soporte tecnológico para dar continuidad a sus procesos formativos en modalidad no presencial.

Por otra parte, considerando que las competencias docentes en materia de planificación, mediación y evaluación formativa son fundamentales para garantizar el reconocimiento del valor de la diferencia y para abordar adecuadamente los procesos de inclusión, el resultado de algunos estudios dan cuenta que "la formación inicial y continua de profesores en Chile no ha enfatizado ni incentivado el desarrollo de competencias docentes para trabajar con grupos heterogéneos desde una perspectiva inclusiva" (Cornejo, 2019, p.30).

Es importante destacar que, de acuerdo a los compromisos asumidos por Chile en cuanto a garantizar una educación inclusiva y de calidad, es deber del Estado asegurar el acceso a la educación, ya sea mediante clases presenciales o a distancia, resguardando principalmente a los grupos más vulnerables (López, 2014).

Así, la actual crisis sanitaria ha puesto a prueba no solo el desempeño de los/as docentes en cuanto a las demandas por una educación de calidad, sino que ha planteado un profundo desafío para la adecuada implementación de estrategias educativas que den cuenta de los compromisos del Estado y las instituciones educativas en materia de inclusión, principalmente si se considera que la escuela "es el mecanismo más eficaz de legitimación de las desigualdades sociales, [pero] es también la herramienta más poderosa para reducir las desigualdades” (Murillo y Duk, 2020, p.12).

Teniendo en cuenta estas consideraciones, la investigación que se presenta en este artículo se planteó abordar la siguiente pregunta: ¿cuáles son las teorías subjetivas de docentes de un colegio particular subvencionado, que se desempeñan en modalidad no presencial, acerca de la inclusión escolar en el actual contexto de pandemia?

Para responder la pregunta planteada, nos enfocamos en el estudio de las teorías subjetivas de los y las docentes, ya que estas permiten orientar la comprensión e interpretación de su realidad, brindar un marco explicativo a fenómenos de experiencias pasadas, y predecir y sugerir nuevos 
comportamientos. Dichas teorías guían el accionar del sujeto e involucran sentimientos de identidad que le dan un sentido subjetivo a la existencia del individuo (Catalán, 2010). Constituyen, por tanto, un intento por acercarse a las explicaciones que dan los propios sujetos acerca del fenómeno estudiado.

La pertinencia de este problema se sustenta en las demandas que actualmente enfrenta el sistema educativo, las cuales toman vida en la figura del profesor y la profesora, profesionales altamente implicados/as en la respuesta que la escuela debe dar a los nuevos requerimientos impuestos por la pandemia.

El presente estudio tiene por objetivo develar las teorías subjetivas que poseen las y los docentes de un colegio particular subvencionado, que se desempeñan bajo modalidad no presencial, en torno a la situación que experimenta la inclusión escolar en contexto de pandemia. En lo específico, y como pasos necesarios para responder al objetivo principal, se busca lo siguiente: a) analizar las teorías subjetivas que sostienen los y las docentes sobre la inclusión escolar; b) describir las teorías subjetivas docentes respecto al impacto de la modalidad no presencial en la calidad del desempeño docente; c) describir los principales aprendizajes docentes en modalidad de educación no presencial; y d) analizar las teorías subjetivas docentes respecto a los desafíos que ha presentado la modalidad no presencial para la inclusión escolar.

De esta forma, se espera que los resultados de esta investigación aporten información relevante para una adecuada intervención en futuras instancias de desarrollo docente, tanto a nivel de formación continua como inicial. Asimismo, se busca aportar al mejoramiento de las prácticas docentes con información actualizada, pertinente y, lo más importante, fundada en las experiencias y elaboraciones de quienes se encuentran directamente involucrados/as en los procesos educativos.

\section{Marco teórico}

\section{Inclusión como derecho a la educación}

El término inclusión se vincula a la disposición y calidad de las interacciones sociales, "a las emociones, a la afectividad y se relaciona con la capacidad de las personas de aceptar al otro y convivir en armonía aceptando las diferencias” (Ávila et al., 2017, p.42). La inclusión, entonces, 
abarca todas las áreas de la sociedad, por lo que implementar comunidades educativas inclusivas se constituye en uno de los grandes desafíos de la educación actual.

Hacer una definición precisa del concepto de inclusión educativa resulta complejo, puesto que no existe consenso al respecto. Sin embargo, el significado que se acerca con mayor fuerza a la perspectiva que asumimos en este estudio es el que señala que "la educación inclusiva y la atención a la diversidad no se refieren a cómo se educa a un grupo especial de alumnos, sino a cómo se educa a todos ellos" (Toboso et al., 2012, como se citó en Sánchez-Teruel et al., 2013). De acuerdo a lo anterior, existe un creciente consenso internacional en torno a asociar la inclusión a la presencia, participación y éxito de todos/as los/as estudiantes, superando así la idea de que únicamente se vincula a estudiantes con discapacidad o necesidades educativas especiales.

De esta manera, compartimos la idea de inclusión educativa como un apoyo a todos/as los/as estudiantes, tal como se prescribe en diversos tratados internacionales que la refieren como un derecho. La Unesco (Organización de las Naciones Unidas para la Educación, la Ciencia y la Cultura, 2009) considera la educación inclusiva como una estrategia clave para lograr los objetivos del proyecto "Educación para Todos", en el entendido de que esta sería un derecho humano básico y fundamental para alcanzar una sociedad más justa e igualitaria. De hecho, en 2015, el Foro Mundial sobre Educación de la ONU (realizado en Incheon, Corea del Sur) planteó como objetivo al año 2030 "garantizar una educación inclusiva y equitativa de calidad y promover oportunidades de aprendizaje permanente para todos" (Unesco, 2015, p.18). En este plano, la apelación a la educación inclusiva como derecho buscaría garantizar una educación de calidad con equidad para todos los/as niños/as y jóvenes en edad escolar.

Así, la inclusión como componente del derecho a la educación, se entiende como un principio rector que orienta el curso de las políticas y prácticas educativas, teniendo como eje el reconocimiento y valoración de la diversidad humana (Murillo y Duk, 2016). Con el fin de reducir la exclusión, por lo tanto, es fundamental identificar y responder a las necesidades de todos/as los/as estudiantes, lo que obliga a una mayor participación de los/as docentes en experiencias que aseguren una integración adecuada, en relación a la atención de las particularidades de las diversas culturas y comunidades. De esta forma, la escuela cumple una función fundamental en torno a la inclusión, puesto que es considerada como garante de dicho derecho. 
En la modalidad no presencial, la inclusión como derecho representa un desafío, debido a que las condiciones pueden hacer difícil garantizar una educación para todos/as los/as estudiantes. Por otra parte, Tarabini (2020) señala que el confinamiento ha puesto de manifiesto las múltiples brechas que posee nuestro sistema educativo, las cuales sitúan a unos colectivos sociales en situación de mayor desventaja escolar. De esta manera, se identifican problemas para asegurar el acceso igualitario y se ve mermada la calidad requerida para que participe y aprenda el estudiantado en su totalidad.

\section{Calidad de la educación e inclusión}

En materia de inclusión, el concepto de calidad de la educación emerge con fuerza, ya que se considera imprescindible para garantizar la inserción adecuada de los/as estudiantes, considerando las demandas y diferencias que presentan los contextos educativos, especialmente en materia de prioridades a nivel estratégico. En términos de su significado y evolución, Aguerrondo (1993) señala que el concepto de calidad de la educación es totalizante, multidimensional y que no permite una reducción únicamente al acto de integrar, sino que implica un acompañamiento permanente de los/as estudiantes, con el fin de asegurar el alcance de los objetivos de aprendizaje bajo un enfoque centrado en la equidad. En opinión del autor, la inclusión se presenta como un medio adecuado para evaluar la eficacia de los sistemas educativos.

Si bien se han llevado adelante políticas educativas que permiten mejorar el acceso universal a la educación y optimizar así su calidad y equidad, aún se presentan notables diferencias educativas. En este aspecto, como señala Tarabini (2020), la educación implica garantizar la transmisión y adquisición de conocimientos profundos y relevantes para todos/as los/as estudiantes, lo que se ve amenazado por las formas de segregación, desvinculación y expulsión que exhibe nuestro sistema educativo, las cuales se dirigen especialmente a los colectivos sociales más vulnerables.

En Chile, durante los últimos años, ha surgido una serie de cuerpos legales orientados a mejorar la calidad de la educación. Entre ellos se encuentra la Ley 20.529 de 2011, que establece la creación y regulación del Sistema Nacional de Aseguramiento de la Calidad de la Educación Parvularia, Básica y Media. Este cuerpo legal busca proveer oportunidades de desarrollo e integración social al conjunto de niños, niñas, jóvenes y personas adultas, de manera equitativa e inclusiva, 
previniendo la discriminación y la segregación de cualquier tipo, garantizando que todas y todos puedan ser ciudadanos/as autónomos/as, responsables, proactivos/as y críticos/as.

Cabe destacar que, en los últimos años, el sistema educativo chileno ha estado afectado por una serie de cambios, vinculados a la necesidad de entregar una educación de calidad que aporte al aprendizaje de todos/as los/as estudiantes. Es así como las políticas de los últimos años han buscado enfocar los cambios "no ya en los resultados, sino más bien en la necesidad de repensar el proceso educativo, buscando su adecuación óptima a los nuevos tiempos y contextos que se dan en los centros educativos" (Viñals y Cuenca, 2016, p.104).

En contexto de pandemia y educación remota de emergencia, se hace necesario observar con detenimiento el proceso educativo, debido al desafío que implica desplazarse desde una modalidad tradicional, ya conocida por docentes y estudiantes, a otra que es no presencial y desconocida por la gran mayoría de ellos/as. Se debe verificar la calidad de la educación implementada, ya que no todos/as los/as estudiantes cuentan con la tecnología ni las condiciones adecuadas para acceder y participar en las clases virtuales. Murillo y Duck (2020) llaman la atención sobre el hecho de que la educación a distancia es una alternativa para quienes tienen equipos de calidad y acceso a internet, pero desgraciadamente hay muchos/as estudiantes que no cuentan con esos recursos ni las condiciones para beneficiarse de esta opción.

En definitiva, si la calidad y equidad educativa se muestran como un enorme reto en la educación tradicional, lo son aún más en la educación no presencial. Ante los desafíos impuestos por la pandemia, el sistema educativo ha debido adecuarse a las nuevas condiciones para procurar garantizar la educación a todo el alumnado. Los y las docentes han cumplido un rol clave a este respecto, ya que han debido replantear sus prácticas educativas y fortalecer sus competencias docentes para lograr dicho objetivo.

\section{Desempeño profesional docente en educación no presencial}

El desempeño profesional docente es un tema de creciente interés a nivel de políticas educativas, puesto que se vislumbra como el principal factor de aseguramiento de la calidad y la inclusión. 
Compartimos la conceptualización de Añorga (2014), quien señala que el desempeño profesional dice relación con la capacidad para llevar a cabo acciones, deberes y obligaciones propias de un cargo, atendiendo las funciones profesionales que exige un determinado puesto de trabajo, vinculadas a la conducta real del profesional y en relación con otras tareas a cumplir.

En Chile, en materia de estandarización, el Marco para la Buena Enseñanza se ha constituido en un factor determinante al momento de proveer orientaciones y criterios acerca de lo que, a nivel ministerial, se considera como un desempeño profesional docente adecuado. La normativa viene así a prescribir aquellos aspectos o criterios que un/a docente debe ponderar para que su ejercicio profesional sea efectivo. Las categorías de desempeño se estructuran en cuatro dominios: preparación del proceso de enseñanza-aprendizaje; creación de un ambiente propicio para el aprendizaje; enseñanza para el aprendizaje de todos/as los/as estudiantes; compromiso con sus responsabilidades y desarrollo profesional (Mineduc, 2008).

Atendiendo el complejo escenario que está enfrentando la educación formal debido a la pandemia por COVID-19, se han generado nuevas exigencias relacionadas con la tarea educativa y, particularmente, con los alcances de la profesión docente, lo que ha obligado a las instituciones educativas a replantear los dominios prescritos por el Marco para la Buena Enseñanza. El cambio desde una educación presencial a una no presencial ha transformado las funciones de la escuela y la totalidad de las acciones relacionadas con el desempeño docente, dificultando la capacidad para cumplir adecuadamente con las exigencias propias de la actividad pedagógica, especialmente en materia de inclusión. A este respecto, una investigación realizada por Cornejo-Valderrama et al. (2017) señala la necesidad de formación continua por parte del profesorado en áreas específicas de la diversidad, referidas al conocimiento y comprensión de aspectos teóricos y la praxis educativa, con objeto de responder adecuadamente a los requerimientos de todo el estudiantado.

La Unesco reconoce que el rol docente es un elemento central en la renovación de las prácticas educativas, promoviendo nuevas maneras de enseñar y nuevas experiencias de aprendizaje, donde la "calidad docente es un factor clave para hacer la conexión entre estudiantes, los dispositivos digitales y el aprendizaje" (Unesco, 2014, p.21). Asimismo, de acuerdo a la Unesco (2015), las TIC plantean al profesorado el gran reto de modificar las estructuras del aprendizaje, sustituyendo una pedagogía basada en la recepción y la repetición, por otra orientada a aprender creando. 
Atendiendo aquello y considerando el impacto que pudiese tener la actual pandemia en el ejercicio docente, la educación no presencial se presenta como un reto importante en términos de asegurar el logro de los aprendizajes esperados, especialmente en el marco de un conjunto de normativas, nacionales e internacionales, que han destacado la inclusión como uno de los medios más adecuados para alcanzar una sociedad más justa.

Cabe destacar que el replanteamiento de las prácticas educativas para el logro de aprendizajes del alumnado, ha permitido a los/as docentes elaborar sus propias teorías con respecto a su desempeño; por lo tanto, se hace indispensable conocer estas creencias para apoyar su labor.

\section{Teorías subjetivas}

Para Flick (2004), las teorías subjetivas son hipótesis que surgen en la vida cotidiana, siendo elaboradas por los sujetos con respecto a ellos mismos y el mundo, estando relacionadas entre sí por su temática. Es decir, se trata de interpretaciones del sujeto acerca de sí mismo y su contexto, las cuales "tienen una estructura argumentativa hipotética, por lo menos parcialmente explícita o explicitable" y orientan el comportamiento del individuo (Catalán, 2010, p.55). De acuerdo a estas definiciones, entendemos que las teorías subjetivas se refieren a la elaboración de los sujetos para lograr desenvolverse en el mundo, puesto que le otorgan sentido a su entorno, guían su acción y le permiten explicar(se) eventos sucedidos.

Las teorías subjetivas, como señala Catalán (2010), no poseen una organización formal comparable con la de las teorías científicas, puesto que una parte de sus contenidos suele estar implícito, sin encontrarse en la memoria organizada como un conjunto de proposiciones. Se encuentran como experiencias traducibles en enunciados bastante flexibles, que se ajustan a circunstancias específicas, sin que puedan cumplir con el requisito de la consistencia interna de las teorías científicas.

Las posibilidades de organización del contenido de una teoría subjetiva pueden ser básicamente dos: contenido explícito, cuando está expresado verbalmente y es susceptible de ser verificado en la acción (comportamiento del sujeto), y contenido implícito, cuando está parcialmente enunciado. 
Estos dos tipos de contenidos dan lugar al contenido implícito explicitable, ya sea en forma comunicable por parte del sujeto o deducible de su discurso, y al contenido implícito no explicitable, cuando los contenidos son inaccesibles para el propio sujeto que los sustenta (Catalán, 2010).

De acuerdo al grado de estructuración de la hipótesis, se pueden distinguir tres niveles de análisis: a) nivel preteórico, que corresponde a enunciados que tienen una base inferencial y se asimilan parcialmente a una hipótesis, donde el/la elaborador/a no es consciente de la teoría planteada; b) nivel teórico restringido, que incluye enunciados sobre una base inferencial, asimilables a una estructura formal próxima a una hipótesis; c) nivel teórico, donde se ubican enunciados sobre una base inferencial, organizados y consistentes, que se asimilan a una hipótesis.

\section{Metodología}

La presente investigación utilizó una metodología de tipo cualitativa, cuyo diseño se inscribe en la grounded theory, ya que enfatiza la generación inductiva de teorías, conceptos, hipótesis y proposiciones desde los datos, a través de la recogida y análisis sistemático de éstos (Rodríguez et al., 1996).

La investigación se realizó en la ciudad de Coquimbo (Chile), en un establecimiento educacional de dependencia particular subvencionada, que imparte enseñanza básica y media. Siguiendo los criterios del muestreo teórico, el número de participantes alcanzó un total de 8 docentes, 2 generalistas y 6 de especialidad.

El muestreo corresponde a un tipo no probabilístico y de variación máxima. Los criterios de inclusión de los/as docentes participantes consideró diversidad de género, variedad de asignaturas y cursos en los que se desempeñan, y poseer a lo menos 3 años de experiencia en el ejercicio docente.

Para la recolección de la información se utilizó la entrevista en profundidad, la que fue validada por tres jueces expertos y se desarrolló sobre tres tópicos: prácticas educativas en educación no presencial, competencias docentes para abordar exigencias educativas y apoyo a la diversidad de los/as estudiantes. Luego, contando con la firma de los consentimientos informados, se procedió a 
entrevistar a los/as participantes mediante la plataforma Zoom. Las entrevistas se realizaron entre los meses de julio y septiembre de 2020 .

Siguiendo los procedimientos de análisis propuestos por la teoría fundamentada de Strauss y Corbin (2002), se trabajó con la codificación abierta, axial y selectiva. Las entrevistas fueron codificadas y analizadas utilizando el programa informático Atlas.ti. El único código intencionado a priori fue la teoría subjetiva, donde se agrupó a todas las teorías subjetivas ya identificadas. En el proceso de codificación axial se dio origen a la elaboración de cuatro ejes temáticos, cuyas relaciones internas se basan en las teorías subjetivas de los/as entrevistados/as, que en esta fase alcanzaron mayor integración y parsimonia. Estos ejes temáticos hacen referencia a inclusión, aprendizaje docente, desempeño docente y evaluación de aprendizajes, en modalidad no presencial. Posteriormente, se realizó el proceso de codificación selectiva, en el que emergió la categoría central "desafío docente".

Como una forma de complementar los procedimientos de análisis utilizados, se consideró el grado de elaboración de las teorías subjetivas identificadas, lo que permitió discernir los niveles teóricos alcanzados por los sujetos (teórico, preteórico o teórico restringido).

En todo momento se mantuvieron presentes criterios de rigor científico como es la triangulación de investigadores, realizando el proceso de codificación abierta por separado, para luego revisar la consistencia y modificar los códigos cuando fuese necesario, a partir del consenso de los investigadores. Posteriormente, el proceso de codificación axial y selectiva se realizó en conjunto. También se tuvo presente la descripción detallada del proceso de recogida, análisis e interpretación de los datos y la aplicación de la estrategia de comparación constante, la cual permite revisar y comparar los resultados emergentes con teorías previamente formuladas, dando cuenta de los criterios de consistencia y confirmabilidad o reflexibilidad. Además, se siguieron los criterios éticos de consentimiento informado y confidencialidad.

\section{Resultados}

A partir del proceso de análisis realizado, se identificaron y establecieron las teorías subjetivas de los y las docentes respecto a sus concepciones sobre inclusión en contexto de pandemia. 
La presentación de resultados se organiza en base a la teorización de los/as participantes, de acuerdo a los propósitos de la investigación. De este modo, se da cuenta de cuatro dimensiones que emergen en el análisis: teorías subjetivas sobre inclusión, teorías subjetivas sobre desempeño docente, teorías subjetivas sobre aprendizaje docente y teorías subjetivas sobre evaluación de aprendizajes, en modalidad no presencial.

La Figura 1 da cuenta de las teorías subjetivas de los/as docentes sobre el proceso de inclusión en contexto de pandemia. Destaca la dificultad que percibe el profesorado para brindar la atención requerida a la diversidad de sus estudiantes, lo que vinculan principalmente con el desconocimiento de estrategias de apoyo, incluyendo una deficiente orientación y acompañamiento a este respecto:

Recién este semestre te dicen 'oye, hay que considerar a los alumnos en la situación diferencial', que hay que hacer la evaluación diferenciada (...). Y es como 'acuérdate de hacerlo’, pero nada más. Pero ¿cómo lo hago?, ¿cómo me contacto con ellos?, ¿cómo lo hago con los chicos que no conozco? ¿Cómo? No tengo las herramientas. Entonces te dejan así, como al vacío". (Sujeto 7)

\section{Figura 1}

Teorías subjetivas sobre inclusión en modalidad no presencial

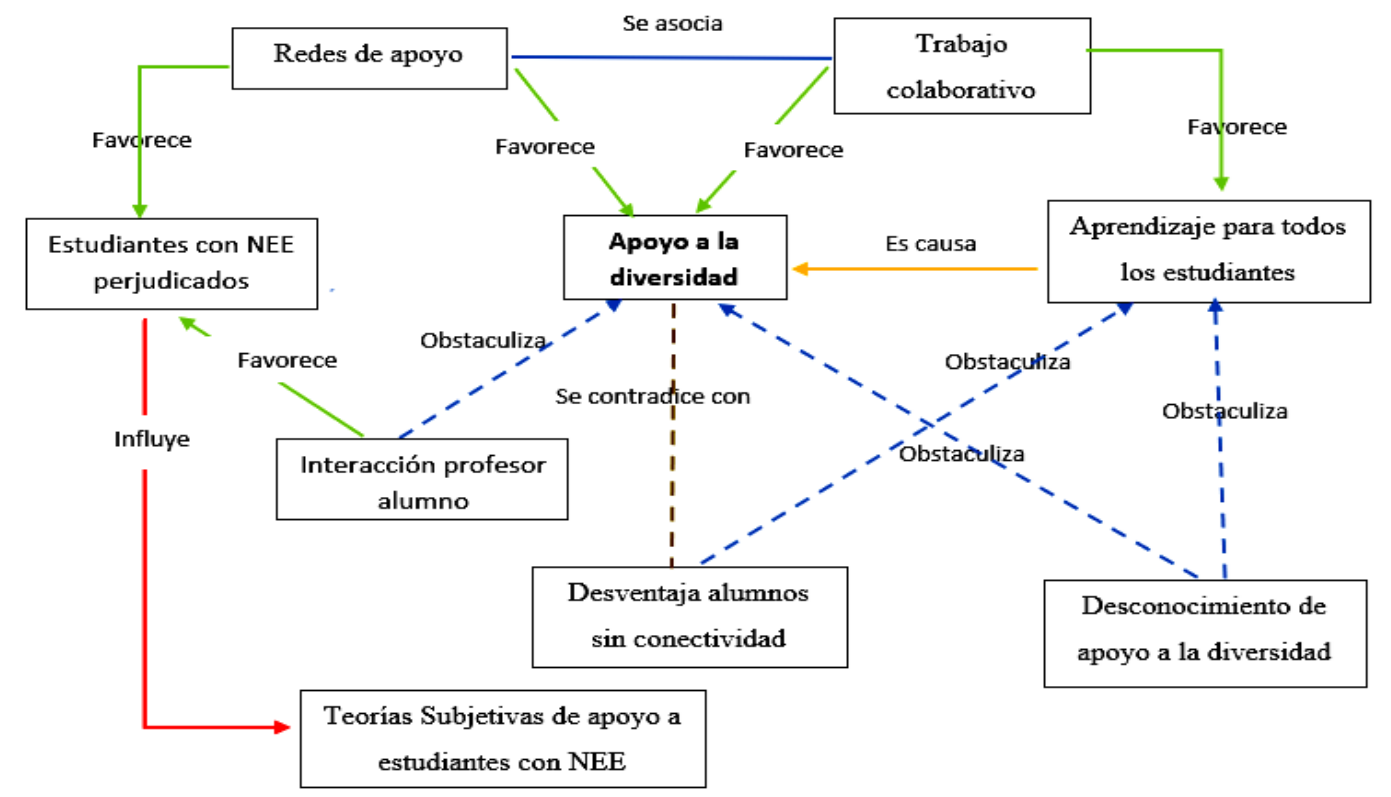


Los y las docentes consideran que los/as estudiantes con necesidades educativas especiales son los/as principales perjudicados/as en el presente contexto educativo, ya que no cuentan con el sustento de profesionales capaces de darles apoyo ni con la presencialidad que los/as docentes visualizan que es necesaria para el progreso académico. Es importante señalar que, para este grupo de docentes, la concepción sobre inclusión y diversidad se restringe a la idea de atención a estudiantes con necesidades educativas especiales, que pertenecen al programa de integración escolar.

A mí me preocupa mucho y pienso que los grandes afectados, perjudicados por esto, son los niños del programa de integración escolar, porque nosotros como profesoras no tenemos las competencias a veces para trabajar con ellos en clase, imagínate las mamás en la casa. (Sujeto 3)

Por otro lado, los y las docentes destacan que las dificultades de conexión que han presentado algunos/as estudiantes los/as ha perjudicado considerablemente, trayendo consigo una notoria desventaja en el ámbito académico y dificultades para consolidar los aprendizajes, tras no contar con la necesaria interacción con el profesorado:

No es lo mismo trabajar con la profesora que hacerlo solo o con la ayuda de los papás, que muchas veces también a ellos les cuesta entender alguna cosa. Por lo tanto, si tienen más dificultades para hacer sus tareas, sus trabajos, sus actividades, no sabemos si están logrando el aprendizaje o solo están entregando la actividad por la nota. Sí, entonces yo creo que están en mucha desventaja. (Sujeto 8)

Los/as docentes creen que existen dos aspectos que favorecen la atención a la diversidad de sus estudiantes: el trabajo colaborativo con otros/as profesionales y las redes de apoyo. Estas últimas favorecerían también la atención de estudiantes con necesidades educativas especiales, quienes enfrentarían las mayores dificultades ante la educación remota, debido al escaso apoyo que pueden recibir en esa modalidad. De este modo, la presencialidad sería fundamental para brindar el acompañamiento necesario, monitorear los avances y corregir lo que fuese necesario en base a las dificultades detectadas: "Presencial puede haber mucho más apoyo, porque estamos ahí, estamos 
al ladito. Porque yo lo estoy viendo, yo lo puedo apoyar. Si no estoy yo, está la educadora. Pero de esta forma es más difícil” (Sujeto 4).

En cuanto a la concepción del desempeño docente en contexto de educación remota (Figura 2), emerge con fuerza la idea de que la priorización curricular permite enfocarse en los objetivos principales, siendo un factor importante para el logro de aprendizajes de los/as estudiantes. La priorización curricular permitiría la adecuación de metodologías e influiría en la flexibilización de la enseñanza que se considera relevante, permitiendo establecer estrategias didácticas acordes a la modalidad educativa actual:

Yo he tomado los objetivos de aprendizaje que se nos piden y en base a eso voy adecuando las estrategias y actividades, según los textos de estudio (...). He ido tratando de flexibilizar un poco el asunto, de acuerdo a lo que los niños pueden. (Sujeto2)

\section{Figura 2}

Teorías subjetivas (TS) sobre desempeño docente en modalidad no presencial

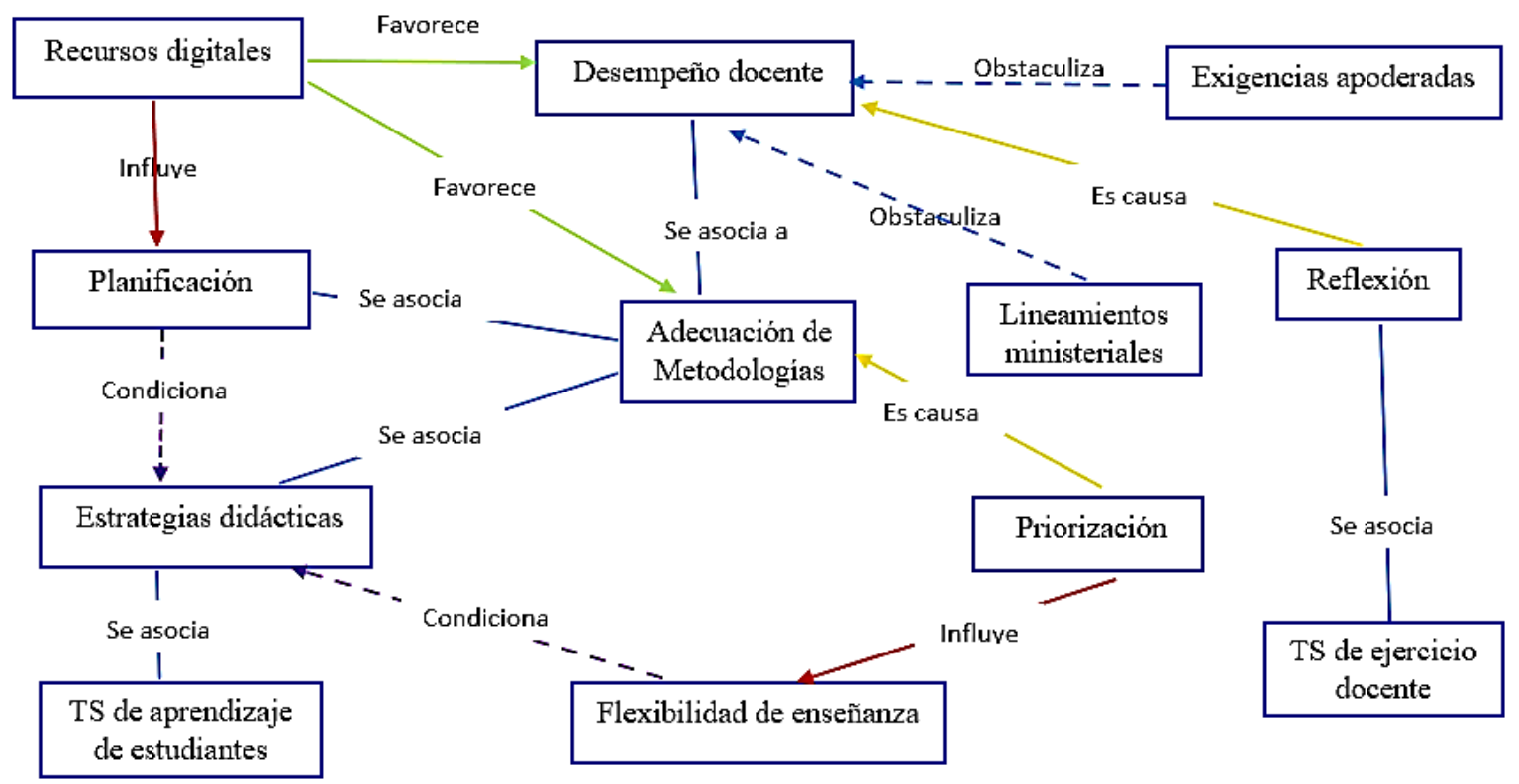


Un aspecto relevante que destacan los/as docentes es la importancia de llevar los aprendizajes a contextos reales y cercanos. Creen que ello puede lograr que los/as estudiantes se motiven, participen y obtengan aprendizajes significativos, ya que lograrían internalizarlos y transferirlos a su realidad:

Yo creo que el aprendizaje, en esta oportunidad que ahora se nos está presentando, en este momento, tiene que ser así, tiene que ser súper cercano, dentro de su entorno. Porque así también va a ser como más real y obviamente les va a quedar con más facilidad, se va a fijar en él con más facilidad. (Sujeto 4)

Otro aspecto que teoriza este grupo de docentes es la relevancia de los procesos reflexivos para desplegar las habilidades y destrezas necesarias al momento de enfrentar los desafíos de la enseñanza y el aprendizaje en contexto de pandemia:

Este tiempo a uno le va mostrando, le va haciendo buscar cuáles son verdaderamente los caminos, los caminos que sean como más certeros, para lograr llegar a los niños, al interés de los niños. Y obviamente, luego tener un aprendizaje en ellos, todo esto se da solamente estando así, porque en un año normal nosotros no nos detenemos mucho, sino que actuamos un poco por inercia. (Sujeto 4 )

Por otro lado, los y las docentes destacan dos aspectos que obstaculizan su desempeño docente: los lineamientos ministeriales y las demandas de los/as apoderados/as. Respecto a lo primero, sus teorizaciones asocian la poca claridad en las orientaciones con imprecisiones cometidas durante su práctica educativa:

Creo que el ministerio fue improvisando, entre comillas, y eso también afectó un poco este proceso porque nunca tuvimos una línea clara. Pero el ministro dijo 'vamos a entrar en abril', después el tema se fue corriendo. Al final entramos en estas cuarentenas totales y al final hemos tratado de irnos adaptando a nuestras metodologías, a nuestras planificaciones, de acuerdo a lo que se ha ido generando con el tiempo. Y el colegio lógicamente va siguiendo las directrices del Ministerio de Educación. Entonces, creo que de ahí ha habido algunos problemas, de ahí parte la base de los errores que tal vez se han ido cometiendo. (Sujeto 5) 
Por otra parte, las exigencias de los apoderados, en general, habrían dificultado de manera considerable el quehacer docente, provocando un desgaste adicional que los/as desmotiva:

No hay como retribución, tampoco por parte de los apoderados, y entonces uno se está desgastando demasiado. Porque en el caso de colocarse en el lugar de uno, hacen todo lo contrario, y eso para uno igual es fome, aunque uno no busca la aceptación de todos. Pero uno lo hace por los niños, pero igual es fome. Por último, que no digan nada, pero que estén cuestionando a cada rato, a cada rato, entonces eso para uno es fome y lamentablemente uno se desmotiva. (Sujeto 3)

La Figura 3 da cuenta de las teorías subjetivas de profesores y profesoras respecto a los aprendizajes docentes en contexto de educación remota. Dicho contexto es visto como un desafío importante, debido al dominio de recursos tecnológicos que es exigido por la educación a distancia. Esta situación que genera agobio, resistencia al cambio y sentimientos de malestar: "Yo soy igual negada para la tecnología. Yo era feliz con mi papel mantequilla, con mi papel kraft (risas), la pizarra y los plumones. Yo soy feliz con eso. Me carga a mí esta cuestión” (Sujeto 6).

\section{Figura 3}

Teorías subjetivas sobre aprendizaje docente en modalidad no presencial

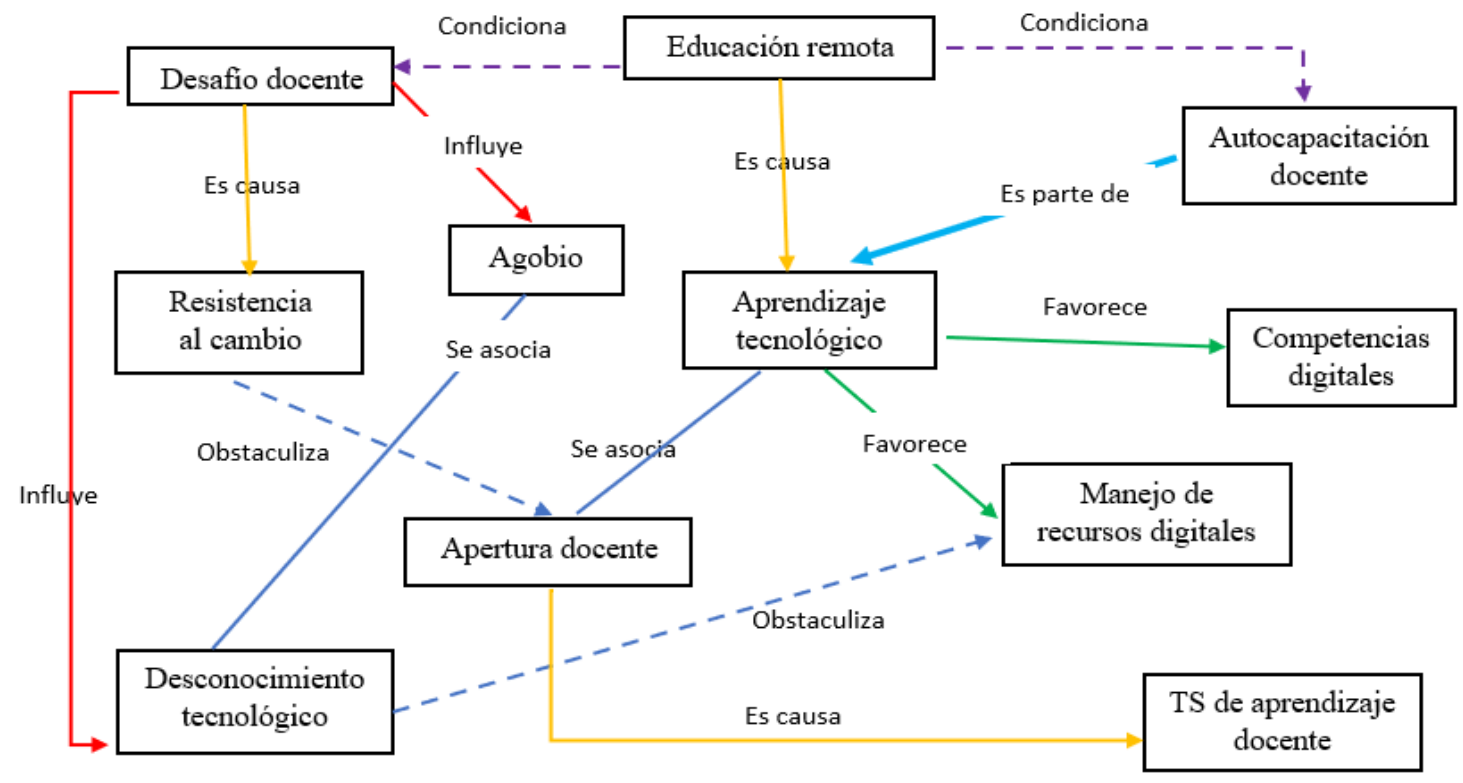


Sin embargo, las demandas del nuevo escenario de enseñanza fueron resueltas a través de la autocapacitación, generando así un aprendizaje tecnológico que las y los docentes asocian con una apertura de sí mismas/os como docentes, lo cual impacta en sus teorizaciones respecto al proceso de enseñanza y aprendizaje:

Creo que es una buena modalidad, ha servido como un incentivo para generar nuevas expectativas; creo que nos ha servido también para aprender una modalidad distinta de trabajo y, a pesar de todo, creo que en el futuro puede generar acá un elemento importante en lo que es la enseñanza-aprendizaje y fortalecernos en ciertas áreas. (Sujeto 1)

Esta apertura a nuevas formas de enseñar y aprender es concebida como una oportunidad para mejorar el ejercicio docente, visualizándose además la permanencia del uso de estos nuevos recursos: "Pensarlas como un aliado porque, si lo ven, estas herramientas las vamos a seguir usando, no van a quedar en el olvido. Cuando volvamos a clases presenciales yo creo que las vamos a utilizar" (Sujeto 2).

Es importante destacar que esta dimensión emerge con fuerza en las entrevistas realizadas, quizás porque es la que ha provocado los mayores cambios personales en los/as docentes. Sus teorizaciones en esta dimensión estuvieron más centradas en sus propias experiencias que en los procesos de inclusión de los/as estudiantes. Sin embargo, nos parece relevante incorporarla precisamente por la intensidad con que aparece y porque podría dar pistas sobre cómo diseñar una estrategia que permita aprendizajes con la misma intensidad, en atención a la diversidad de los/as estudiantes.

La Figura 4 hace referencia a las concepciones de los/as docentes respecto a la evaluación en modalidad no presencial. Profesores y profesoras teorizan principalmente sobre la relación entre la evaluación en el actual contexto y la incertidumbre sobre el logro de aprendizajes, dadas las dificultades para verificar estos últimos, el apoyo excesivo de los/as apoderados/as y el ausentismo escolar: 
Yo creo que eso va a ser un tremendo desafío, el poder evaluar de buena manera... Que esto no sea subjetivo porque, como le dije, si me llega una guía muy bien hecha, a lo mejor no fue hecha por el niño. (Sujeto 2)

\section{Figura 4}

Teorías subjetivas sobre evaluación de aprendizajes en modalidad no presencial

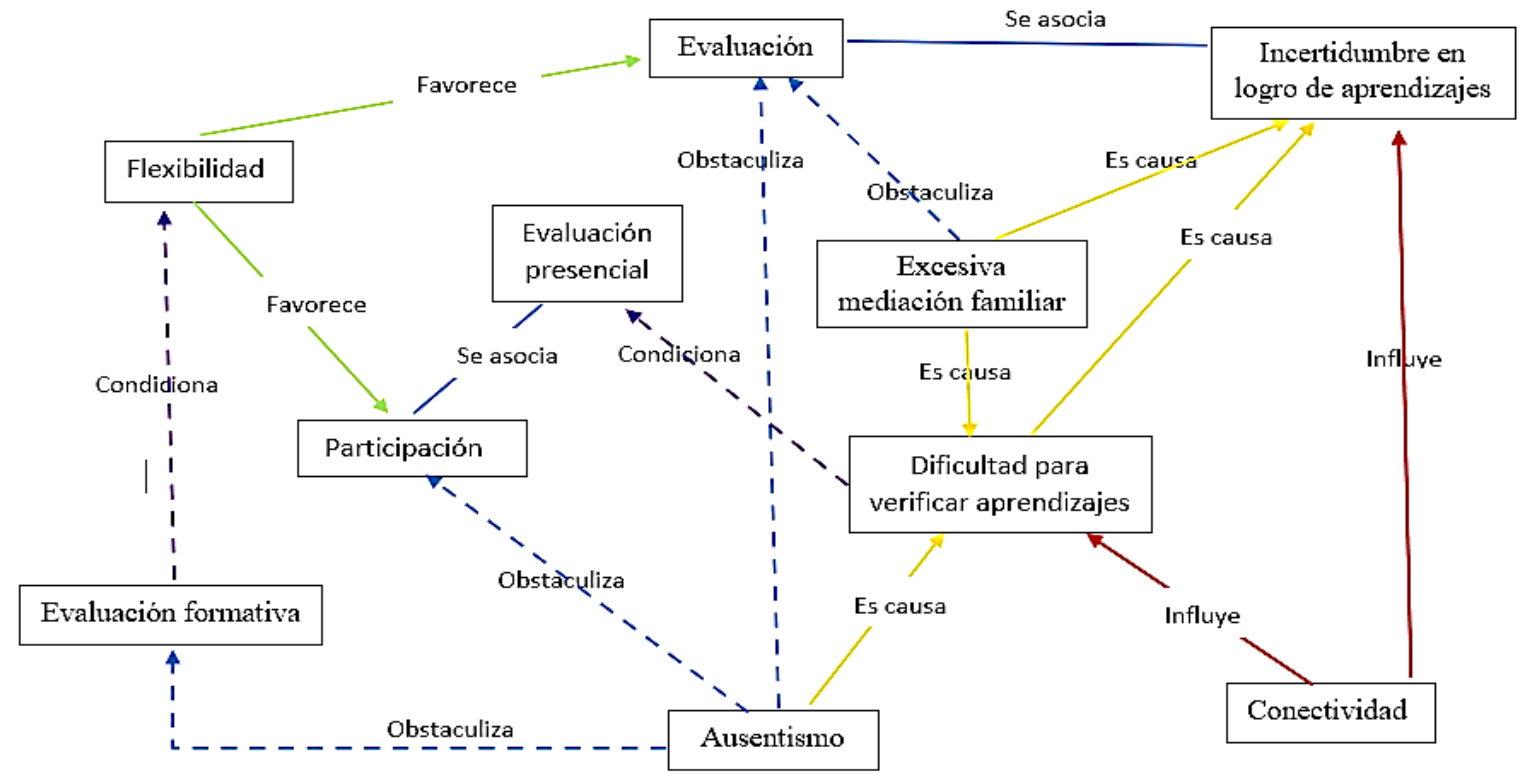

Los/as docentes entrevistados/as plantean que, para corroborar los aprendizajes, se debe realizar una evaluación presencial que permita observar directamente el proceso realizado por los y las estudiantes. Por otra parte, flexibilizar el proceso evaluativo implicaría realizar las evaluaciones de manera formativa, mediante una relación que involucre al docente y el/la estudiante, utilizando la técnica de preguntar como estrategia para acceder a los conocimientos del estudiantado:

La evaluación va netamente de manera formativa, en preguntar constantemente dentro de los procesos de clase online. Una interacción entre el profesor, que soy yo, y el alumno, para ver si va aprendiendo a través de preguntas, a través de desarrollo. Por ejemplo, de las distintas temáticas que estamos viendo, el alumno opina, o sea, alguna participación. (Sujeto 1) 
De acuerdo a los hallazgos de la investigación y del proceso interpretativo efectuado, emerge como categoría central "desafío docente", ya que permite integrar y dar sentido a las teorías subjetivas de los sujetos de investigación. Dicha categoría hace mención a los diversos retos que la educación no presencial ha planteado a los/as docentes para hacer posible la inclusión de sus estudiantes y adaptarse a los requerimientos del actual contexto educativo, donde han debido adecuar las formas tradicionales de educar.

Así, el desafío docente que emerge con mayor frecuencia se relaciona con el aprendizaje tecnológico. Para la mayoría de los/as docentes entrevistados/as el uso de los recursos digitales fue un reto importante, ya que no contaban con las competencias necesarias para impartir sus clases en modalidad remota. En esta línea, los y las docentes consideran que han tenido un aprendizaje meritorio, el cual les ha permitido desenvolverse en el nuevo escenario educativo, enriquecer su ejercicio docente y fortalecer habilidades que no habían desarrollado hasta ese momento. Este desafío docente lo teorizan en torno a la experiencia educativa en general y no como un aspecto específico vinculado a la inclusión de los estudiantes.

A pesar de estos aspectos positivos, el desempeño docente es percibido como un desafío. Las decisiones de las autoridades son consideradas erráticas, pues solicitan desarrollar una nueva modalidad de trabajo sin que el profesorado cuente con las orientaciones y las capacitaciones adecuadas. Todo ello provocaría agobio y resistencia al cambio, más aún para el proceso de inclusión.

Respecto a la atención a la diversidad del estudiantado, los/as docentes señalan tener grandes dificultades, las cuales también se presentaban en contexto de presencialidad. Expresan que no han recibido formación especializada para atender a estudiantes con necesidades educativas especiales y que no cuentan con las herramientas necesarias para apoyarlos/as con las adecuaciones que requieren, ya sea en el actual contexto remoto o en modalidad presencial.

Respecto a la atención a la diversidad y su fundamento esencial, que es el aprendizaje de todos/as los/as estudiantes, los/as docentes consideran que es un desafío y un ideal por parte de las autoridades educativas. Expresan que la realidad presente en las aulas no permite que se consoliden los aprendizajes en la totalidad del alumnado, mucho menos si la enseñanza se desarrolla en 
modalidad no presencial. Un punto a destacar es que los/as docentes vinculan esta imposibilidad a factores que son externos a sus competencias docentes. En este sentido, los problemas de conectividad por parte del estudiantado son considerados un desafío relevante y una falencia del sistema que obstaculiza la atención a la diversidad. Por tratarse de un factor sobre el cual los/as docentes no tienen control, se trata de un obstáculo de difícil solución.

Por otra parte, la flexibilidad es considerada como un aspecto importante en el apoyo a la diversidad. Adecuar la metodología y las estrategias de enseñanza al contexto educativo imperante permitiría favorecer el aprendizaje del estudiantado, especialmente de quienes presentan mayor dificultad para participar y comprender los contenidos durante las clases virtuales.

\section{Figura 5}

Teorías subjetivas docentes acerca de la inclusión escolar en contexto de pandemia

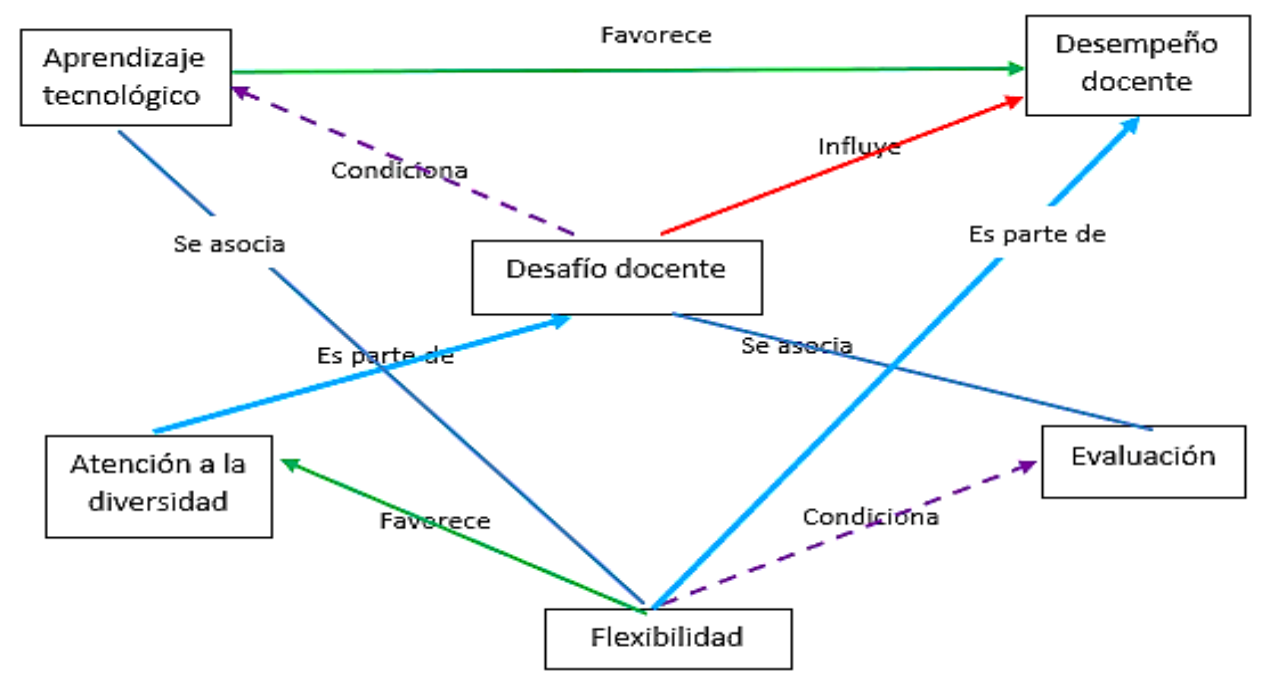

El desafío de la modalidad no presencial también se asocia a la evaluación. Según lo destacado por el profesorado, existe incertidumbre respecto al logro de aprendizajes por parte de los/as estudiantes, dada la excesiva mediación de los/as apoderados/as y al ausentismo que se ha manifestado en las clases virtuales, lo cual plantea la necesidad de corroborar los aprendizajes mediante evaluaciones presenciales. Esto ha influido en el ejercicio docente, haciendo necesario 
modificar el concepto tradicional de evaluación para adecuarse al contexto y las necesidades de los/as estudiantes que han presentado dificultades de acceso.

Los hallazgos de la investigación sugieren que el nivel de estructuración de las teorías subjetivas docentes respecto a la inclusión y la atención a la diversidad, en su mayoría, aún se encuentran en un nivel preteórico o teórico restringido. Esto da cuenta de una falta de reflexión personal en torno a temáticas que se están abordando hace varios años en el sistema educativo chileno. Lo anterior permitiría comprender por qué la inclusión y la atención a la diversidad son percibidas como un desafío, incluso anterior al contexto de pandemia, y por qué se sigue señalando que no se cuenta con las competencias profesionales necesarias para el proceso de inclusión escolar.

\section{Discusión}

En base al proceso de interpretación de las teorías subjetivas, se identifican las principales concepciones sustentadas por los y las docentes respecto a la situación que experimenta la inclusión escolar en contexto de pandemia, así como el desempeño docente bajo modalidad no presencial.

Con respecto a las teorías subjetivas docentes sobre inclusión, la idea que surgió con mayor frecuencia fue la limitación que expresan tener profesores y profesoras al momento de atender la diversidad del estudiantado, restringiendo dicho concepto a niños y niñas que son parte del programa de integración escolar y que presentan necesidades educativas especiales. Los/as docentes señalan que no cuentan con las herramientas para enseñar y brindar el acompañamiento necesario a estos/as estudiantes. Expresan, además, que esa falta de preparación ya se daba antes, en contexto de presencialidad, y que se acrecienta ahora en la modalidad remota. Estas teorías subjetivas revelan, tal como visualizan Cornejo-Valderrama et al. (2017), que existe un desafío en materia de formación continua en áreas específicas de la diversidad, referidas al conocimiento y comprensión de aspectos teóricos y la praxis educativa, para responder de manera adecuada a los requerimientos de todo el estudiantado. Los y las docentes perciben esta situación como una barrera y una exigencia de mayor esfuerzo de su parte, señalando además la ausencia de redes que ayuden a ejecutar esta función. Asimismo, reconocen que los/as estudiantes con dificultades son los/as principales perjudicados/as, ya que no reciben el apoyo requerido en el presente contexto 
educativo, así como tampoco lo hacían en el pasado. Este hallazgo emerge como un desafío para responder al principio de equidad y brindar una educación de calidad para todos y todas, en especial para quienes se encuentran en mayor riesgo de exclusión.

Por otra parte, las y las docentes señalan que las dificultades de conexión han perjudicado considerablemente a ciertos/as estudiantes, quienes han experimentado una notoria desventaja en su proceso formativo. Reconocen que no existe igualdad de oportunidades al momento de participar en las clases virtuales, concordando así con lo planteado por Tarabini (2020), quien sostiene que el confinamiento ha revelado las múltiples brechas que presenta nuestro sistema educativo, las cuales sitúan a unos colectivos sociales en situación de mayor desventaja escolar. Estas teorías subjetivas dan cuenta del reto de nuestro sistema educativo para alcanzar el objetivo establecido en el Foro Mundial sobre Educación de la ONU realizado en 2015, donde se planteó que al año 2030 debería garantizarse una educación inclusiva, equitativa y de calidad, y promoverse oportunidades de aprendizaje permanente para todos y todas. Ir en pos de este objetivo supone garantizar el acceso de los/as estudiantes a la educación no presencial, lo que es imperioso de lograr en contexto de pandemia.

En cuanto a las teorías subjetivas sobre el aprendizaje docente en contexto de educación no presencial, existe consenso en señalar que los conocimientos adquiridos impactan positivamente en la calidad de la labor docente. El aprendizaje docente logrado se percibe como una apertura a nuevas estrategias de enseñanza, las cuales contrastan con las que son utilizadas en la educación presencial tradicional. De esta manera, se percibe un fortalecimiento de la propia práctica docente, lo que emerge como una oportunidad para enfrentar de mejor manera las demandas de la educación actual. Sin embargo, las/os docentes participantes no establecen una relación entre estos aprendizajes y la posibilidad de brindar una mayor y mejor atención a todos/as sus estudiantes, lo cual se puede comprender en el entendido de que visualizan la inclusión escolar como un problema difícil de resolver, incluso en presencialidad, el cual se vuelve imposible de manejar en contexto de educación remota.

Considerando que el nivel de estructuración de las teorías subjetivas de los/as docentes respecto a la inclusión y la atención a la diversidad se encuentran, en su mayoría, en un nivel preteórico o teórico restringido, se puede establecer que es necesario desarrollar un trabajo focalizado e intencionado con los y las docentes para lograr un cambio en sus teorías subjetivas actuales. 


\section{Conclusiones}

La presente investigación da cuenta de las teorías subjetivas reveladas por las/os profesores/es participantes, con relación a la inclusión escolar y el desempeño docente en contex to de educación no presencial. Con respecto a las teorías subjetivas acerca de la inclusión, se puede señalar que estas se sostienen en la creencia de que existen dos grandes obstaculizadores para la atención a la diversidad en contexto de educación remota. Por un lado, se encuentran las dificultades para el acceso y uso de plataformas digitales por parte de ciertos/as estudiantes y sus familias, lo cual aparece como una falencia de la presente modalidad educativa que incide en la exclusión de ciertos estudiantes. Por otro lado, se encuentran la falta de herramientas del profesorado para apoyar la diversidad y la falta de orientaciones para desempeñarse en este contexto, lo cual los/as deja en una situación de "vacío", donde no logran hallar las estrategias para realizar las adecuaciones que ciertos/as estudiantes necesitan.

Si bien consideran que estos factores dificultan la participación de los/as estudiantes, estiman también que la distancia física influye en la incapacidad de aplicar estrategias más personalizadas, ya que la virtualidad impide la interacción requerida entre docentes y estudiantes con discapacidad o problemas de aprendizaje.

Respecto a las teorías subjetivas sobre el desempeño docente en modalidad no presencial, resulta interesante constatar que, si bien el nuevo contexto significó un gran desafío y causó agobio, malestar y resistencia ante las nuevas exigencias que trajo consigo, al mismo tiempo, las y los docentes conciben los nuevos conocimientos adquiridos como un fortalecimiento de su ejercicio docente y como un beneficio para el desempeño de su práctica educativa. Además, vislumbran que la enseñanza mediada por tecnologías llegó para quedarse.

Por último, podemos señalar que la actual catástrofe sanitaria y su impacto en la educación formal ha puesto a prueba no solo el desempeño docente-y la necesidad de cambio de sus teorías subjetivas actuales, sino que también ha planteado un profundo desafío para la adecuada implementación de estrategias educativas que den cuenta de los compromisos de las instituciones en materia de inclusión. Lo anterior, considerando la importancia de un trabajo pedagógico que se 
adecúe a los requerimientos y características de todos/as los/as estudiantes y que, a partir de ello, garantice su aprendizaje y permanencia. Claramente, la pandemia ha generado una serie de interrogantes en términos de la concreción de estos postulados, especialmente en un contexto que, a la fecha, aún se presenta como incierto respecto de sus repercusiones. La modalidad remota pareciera poner a prueba no solo las competencias de los y las docentes, sino al sistema educativo en su totalidad.

\section{Referencias}

Aguerrondo, I. (1993). La calidad de la evaluación: ejes para su definición y evaluación. Revista Iberamericana de Desarrollo Educativo, 37(116), 561-578. https://tallereduca.files.wordpress.com/2014/07/aguerrondo-calidad-educacic3b3nejes.pdf

Añorga, J. (2014). La educación avanzada y el mejoramiento profesional y humano. Revista Varona, (58), 19-31. https://www.redalyc.org/pdf/3606/360634165003.pdf

Ávila, C., Rodríguez, V. y Hernández, C. (2017). La inclusión escolar de los niños con necesidades especiales. [Tesis de maestría no publicada]. Universidad de La Laguna. https://riull.ull.es/xmlui/handle/915/5727.

Booth, T. y Ainscow, M. (2015). Guía para la educación inclusiva. Desarrollando el aprendizaje y la participación de los centros escolares. OEI.

Catalán, J. (2010). Teorías subjetivas, aspectos teóricos y prácticos. Universidad de La Serena.

Cornejo, J. (2019). Inclusión educativa desde la óptica de los académicos responsables de la formación de profesores de la Universidad Católica del Maule - Chile. Revista de Educación, 28(55), 27-58. https://doi.org/10.18800/educacion.201902.002 
Cornejo-Valderrama, C., Olivera-Rivera, E., Lepe-Martínez, N. y Vidal-Espinoza, R. (2017). Percepción de los agentes educativos respecto de la atención a la diversidad en establecimientos educativos. Revista Electrónica Educare, 21(3), 327-350. https://dialnet.unirioja.es/servlet/articulo?codigo $=6114888$

Flick, U. (2004). Introducción a la investigación cualitativa. Editorial Morata.

Garrido, M. (2020). Educar en tiempos de pandemia: acentuación de las desigualdades en el sistema educativo chileno. Revista Caminhos da Educacao: diálogos, culturas e diversidades. 2(2), p. 43-68. https://doi.org/10.26694/caedu.v3i1.11241

Ley 20.529. Sistema Nacional de Aseguramiento de la Calidad de la Educación Parvularia, Básica y Media y su Fiscalización. Publicada en el Diario Oficial de la República de Chile, 27 de agosto de 2011 .

López, N. (2014). Mercado o garantía de derechos. Modelos en debate para la educación escolar. https://www.unicef.org/chile/media/1411/file/mercadoogarantia_de_derechos.pdf

Mineduc (2020a). Orientaciones Covid-19. https://www.mineduc.cl/wpcontent/uploads/sites/19/2020/03/OrientacionesMineduc_CO VID19.pdf

Mineduc (2020b). Orientaciones para docentes y equipos directivos para guiar el aprendizaje de los estudiantes a distancia. https://bibliotecadigital.mineduc.cl/handle/20.500.12365/17209

Mineduc (2008). Marco para la Buena Enseñanza. https://www.docentemas.cl/docs/MBE2008.pdf.

Murillo, J. y Duk, C. (2016). La inclusión como dilema. Revista Latinoamericana de Educación Inclusiva. 10(1), 11-14. http://www.rinace.net/rlei/numeros/vol10-num1/editorial.pdf

Murillo, J. y Duk. C. (2020). El Covid-19 y las brechas educativas. Revista Latinoamericana de Educación Inclusiva. 14(1), 11-13. https://scielo.conicyt.cl/pdf/rlei/v14n1/0718-7378-rlei14-01-11.pdf 
Organización para la Cooperación y el Desarrollo Económicos. (2019). Perspectivas de habilidades en la OCDE 2019: prosperar en un mundo digital. Editorial OCDE. https://doi.org/10.1787/d2a805cf-es

Rodríguez, G., Gil, J. y García, E. (1996). Metodología de la investigación cualitativa. Aljibe.

Sánchez- Teruel, Robles- Bello, M. (2013). Inclusión como clave de una educación para todos. Revisión teórica. Revista Española de Orientación y Psicopedagogía. 24(2), 24-26. https://www.redalyc.org/pdf/3382/338230794003.pdf

Strauss, A. y Corbin, J. (2002). Bases de la investigación cualitativa. Técnicas y procedimientos para desarrollar la teoría fundamentada. Editorial Universidad de Antioquia.

Tarabini, A. (2020) ¿Para qué sirve la escuela? Reflexiones sociológicas en tiempos de pandemia global. Revista de sociología de la educación - RASE, 13(2), 145- 155. https://doi.org/10.7203/RASE13.2.17135.

Organización de las Naciones Unidas para la Educación, la Ciencia y la Cultura. (2009). Directrices sobre políticas de inclusión en la educación. Unesco. http://escolares.ujed.mx/Documentos/Tutorias/08DirectricesPoliticasInclusion(UNESCO2 009).pdf

Organización de las Naciones Unidas para la Educación, la Ciencia y la Cultura. (2014). The right to education: Law and policy review guidelines. Unesco.

Organización de las Naciones Unidas para la Educación, la Ciencia y la Cultura. (2015). Declaración de Incheon y Marco de Acción para la realización del Objetivo de Desarrollo Sostenible 4. https://docplayer.es/32939958-Declaracion-deincheon.html

Viñals, A. y Cuenca, J. (2016). El rol del docente en la era digital. Revista Interuniversitaria de Formación del Profesorado, https://www.redalyc.org/pdf/274/27447325008.pdf 